\title{
e-Migrinter
}

$14 \mid 2016$

L'ajustement méthodologique comme fabrique critique du savoir dans les études migratoires

\section{Sylvie Bredeloup, Migrations d'aventures. Terrains africains}

\section{Bakary Diakhité}

\section{(2) OpenEdition \\ Journals}

Édition électronique

URL : https://journals.openedition.org/e-migrinter/723

DOI : 10.4000/e-migrinter.723

ISSN : 1961-9685

Éditeur

UMR 7301 - Migrinter

Référence électronique

Bakary Diakhité, «Sylvie Bredeloup, Migrations d'aventures. Terrains africains », e-Migrinter [En ligne], 14 | 2016, mis en ligne le, consulté le 20 mai 2021. URL : http://journals.openedition.org/e-migrinter/723 DOI : https://doi.org/10.4000/e-migrinter.723

Ce document a été généré automatiquement le 20 mai 2021.

Tous droits réservés 


\title{
Sylvie Bredeloup, Migrations d'aventures. Terrains africains
}

\author{
Bakary Diakhité
}

\section{RÉFÉRENCE}

Bredeloup, Sylvie (2014) Migrations d'aventures. Terrains africains, Paris, Éditions du Comité des Travaux Historiques et Scientifiques, 141 p. (CTHS,-Géographie ; ${ }^{\circ} 11$ ).

1 Cet ouvrage de Sylvie Bredeloup propose une réflexion générale sur les «migrations d'aventures " à partir des différents terrains, tout en constituant une synthèse de ses travaux sur les migrations. L'auteure interroge les migrations africaines au travers de la notion de "voyages d'aventure ». Elle met ainsi en dialogue différents lieux et diverses figures d'aventuriers dans le but de saisir les itinéraires migratoires, leurs mises en récit et les imaginaires que les migrants construisent. Après avoir dressé le panorama géographique et historique de l'ensemble des espaces concernés par ces mobilités (lieux de transit, lieux d'installation), Sylvie Bredeloup invite à clarifier la notion "d'aventure " en l'érigeant en un véritable outil d'analyse. Elle adopte une démarche privilégiant d'une part, l'analyse de diverses figures africaines de la réussite puisées dans des cas ethnographiques comme dans les épopées (romans et films), et d'autre part, elle mobilise les œuvres de littérature d'aventure et des récits de voyages entrepris par des Européens pour en faire une analyse minutieuse des migrations africaines. L'auteure s'intéresse en particulier à la mise en récit de «l'aventure migratoire » (p. 23). Reprenant les écrits de Jean-Paul Sartre, elle considère que la mise en récit permettrait d'élever un événement ordinaire au rang d'aventure : «J'ai cru qu'on pouvait définir l'aventure comme: un événement qui sort de l'ordinaire, sans être forcément extraordinaire. On parle de la magie des aventures [...]. Il m'est arrivé des histoires, des événements, des incidents, tout ce qu'on voudra. Mais pas des aventures. Ce n'est pas une question de mots; je commence à comprendre [...] : pour que l'événement le plus banal devienne une aventure, il faut et il suffit qu'on se mette à raconter. C'est ce qui dupe les gens : un homme, c'est toujours un conteur d'histoires, il 
vit entouré de ses histoires et des histoires d'autrui, il voit tout ce qui lui arrive à travers elle; et il cherche à vivre sa vie comme s'il la racontait " (Sartre, 1972, pp. 23-24).

2 Ainsi, Sylvie Bredeloup donne du contenu à cette notion « d'aventure migratoire » dans le cas des migrations subsahariennes. Pour ce faire, la géographe réalise un détour historique et retrace les différentes étapes de la migration des populations originaires d'Afrique noire. Elle présente les phases historiques et majeures qui ont marqué le départ de ces hommes et femmes africains motivés par la quête de nouvelles ressources. Des destins rythmés par une tension entre la crainte de l'exil et la soif d'aventures, qui sont autant de représentations de l'expérience des migrants africains. Les trajectoires sociales et géographiques de ces aventuriers, chemins faits d'étapes et d'angoisses, apparaissent comme des lignes de vies marquées par des ruptures successives, des changements de lieux associés à la peur de ne jamais revenir. Aux premiers marins ou «têtes de pont " (une filière militaire qui s'est mise en place dès la première guerre mondiale 1914-1918), considérés par l'auteure comme les véritables pionniers de cette migration africaine, ont succédé différents profils de migrants au fil des époques et des générations. Les changements socio-économiques et politiques intervenus au cours de l'histoire (fin de la Deuxième Guerre mondiale, migration de main d'œuvre à partir des années 1950-1960), ont en effet transformé profondément une migration considérée à ses débuts comme temporaire avec la perspective d'un retour certain au pays d'origine en une migration durable ou définitive marquée par l'arrivée des femmes dans le cadre du regroupement familial. Le séjour se prolonge au fil du temps et les migrants africains, pris au piège de "l'exil », vont planifier leur intégration dans les différentes sociétés d'installation grâce au commerce et à l'industrie. L'auteure analyse cette destinée marquée par la réussite des uns et l'échec des autres, tous inscrits dans une quête perpétuelle de mieux-être et de conditions de vie plus favorables en dehors de leur espace d'origine (anciens marins, étudiants, diamantaires, etc.).

3 L'ouvrage est organisé en neuf chapitres. Dans le premier, l'auteure revient sur les définitions et les représentations de l'aventure et de l'aventurier telles qu'elles ont été construites dans les sociétés européennes, pour mieux en comprendre les éléments structurants et leurs fluctuations au fil du temps. Elles renseignent sur la manière dont les représentations et les désirs, les vertus et les vices qui lui sont associés, se construisent et se déconstruisent (p. 19). Les glissements à l'œuvre entre migration et aventure sont interrogés dans le deuxième et le troisième chapitre. Ces deux chapitres traitent la question de la mobilité spatiale envisagée comme un impensé. Pour l'auteure, tous les migrants ne sont pas des aventuriers. Comme elle le souligne, «si les aventuriers peuvent être immobiles, les migrants, dans leur grande majorité, déploient et organisent leur itinéraire dans le cadre de réseaux divers » (p. 18). C'est ainsi que dans le chapitre quatre, l'auteure examine la place dans l'histoire migratoire africaine des figures pionnières du « jaguar » et du « sapeur ». Le jaguar était devenu l'icône de tous les jeunes colporteurs qui circulaient entre le Soudan, le Niger, la Gold Coast (actuel Ghana) et le Nigéria, non pas sous la contrainte, mais par désir d'aller vers l'inconnu et de s'affranchir de l'ordre coutumier (p. 43). L'aventure migratoire est alors interrogée au regard de la montée de l'individualisation dans le cinquième chapitre. Après une comparaison de divers récits d'aventuriers inscrits à des époques différentes, l'auteure considère, à l'instar de C. Rousseau et alli., que «l'aventurier du XVIIe et 
XVIIIe siècle construit l'individualisme moderne" (p. 55). Sylvie Bredeloup analyse dans le sixième chapitre, l'expérience collective et/ou individuelle de ces aventuriers de la migration. L'aventure est dévoilée comme étant le temps de l'impatience et de l'accélération. «Les anciens diamantaires du fleuve Sénégal, les nouvelles générations de migrants rencontrés par l'auteure dans le quartier de Noailles à Marseille, tout comme les ghettomen d'Abidjan " n'ont pas l'intention d'attendre pour grimper les échelons de la hiérarchie sociale (p.65). Car, d'après Brigitte Bertoncello et Sylvie Bredeloup (2003, p. 65), la « recherche de gain s'opère dans l'urgence, il n'est pas question de progresser par étapes, d'attendre son heure ni d'accumuler du capital: l'emballement est de rigueur, l'impatience, l'enthousiasme et la créativité semblent être les moteurs de ces entrepreneurs d'un nouveau genre ».

4 Dans le chapitre sept, la géographe porte un regard croisé sur les terrains d'aventure crées par les migrants et leurs rapports à ces territoires. Selon elle, «les migrants africains peuvent être considérés comme des acteurs déterminants de la fabrique urbaine, dès lors où, en investissant les lieux traversés, ils contribuent à leur transformation ainsi qu'à l'émergence de nouvelles urbanités » (p. 99). Les imaginaires de ces aventuriers, en fonction des époques, des générations et du degré d'exposition aux médias, sont ensuite abordés dans le huitième chapitre. De plus en plus, les migrations internationales et les médias ont accordé un rôle capital à l'imagination. « Il ne s'agit pas de dire que les sociétés n'avaient pas fait appel par le passé à cette faculté dans leurs productions mythologiques, littéraires ou artistiques, mais plutôt d'avancer l'idée que l'imagination désormais investit et irrigue l'ensemble des pratiques quotidiennes» (Appadurai, 2005, p. 101). Enfin, dans le chapitre neuf, l'auteure examine avec attention le sexe de l'aventure migratoire. Ce dernier chapitre s'extirpe de «l'impensé » (p. 116), à travers la figure de l'aventurière. Elle aborde l'élaboration des stratégies spécifiquement fondées sur le genre afin de tirer profit de l'activité de commerce dans différents lieux (Dubaï, Guangzhou, Marseille et Hong Kong). La migration longtemps pensée et déclinée au masculin, s'est de plus en plus féminisée. Cette féminisation de la migration fait aujourd'hui des femmes «des oiseaux migrateurs» (p. 111). En Afrique par exemple, la complexité des rapports de genre pousse des femmes à emprunter les routes commerciales et migratoires pour trouver une liberté et une reconnaissance peu accessible dans leurs sociétés encore largement dominées par les hommes (p. 130) ${ }^{1}$.

5 L'ouvrage nous plonge au cœur du voyage de ces migrants notamment dans les espaces traversés et travaillés par ceux-ci. La notion d'« espace travaillé » renvoie aux lieux de transit empruntés par les migrants pour gagner le Nord. Ce rapport aux espaces fait dire à l'auteure que l'expérience des aventuriers façonne les lieux qu'ils traversent et dans lesquels ils impriment leurs propres ambitions : forêts fouillées ou parcourues par les diamantaires (p. 89); quartiers de Belsunce et de Noailles à Marseille (p. 95); tchads et ghettos des migrants subsahariens en Libye. Autant de localités auxquelles les migrants impulsent de «nouvelles fonctionnalités » (pp. 96-97). De fait, ces «territoires entièrement scrutés par les migrants » (p. 99) s'ancrent dans l'esprit de ceux qui les traversent et constituent alors une "mémoire collective " (Halbwachs, 1997, p. 99) en migration. Le lieu par excellence où se greffe cette mémoire est celui du ghetto qui, par le biais d'un processus de "re-paysement", se situe alors dans une "démarche résolument cosmopolite ou cosmopolitique » (p. 99). 
6 Dans le souci de situer les différentes représentations produites par les acteurs, ainsi que leurs émotions, Sylvie Bredeloup accorde une place centrale aux imaginaires migratoires. Elle adopte une démarche comparative entre les récits de soi des sapeurs congolais étudiés par Justin-Daniel Gandoulou (1989) et ceux des diamantaires et migrants rencontrés par l'auteure elle-même sur ses différents terrains d'investigation. De par cette mise en comparaison de divers acteurs aux profils a priori distincts, elle fait émerger une attitude commune où, prime la « réalisation de soi » sur les motifs utilitaires. Les sapeurs mettent en scène leur propre élégance (mode vestimentaire), valorisée par rapport aux capacités matérielles de ceux qu'ils appellent les «grands messieurs ", tout comme certains migrants affirment leur « désir d'aventure » (p. 56), au détriment des projets économiques. Cette envie d'ailleurs, analysée à partir de la tension individuel/collectif, correspond à une « recherche » de « valeurs en soi, et non plus seulement autour de soi, dans la famille, dans la société » (p. 55) et conduit à des processus d'«individualisation de fait». Ce désir donne lieu à des parcours émancipatoires, qui n'excluent pas pour autant la mise en œuvre de stratégies visant à atteindre de "nouvelles familles métaphoriques» (de Latour, 2001, p. 2, citée par Bredeloup, 2014, p. 58), comme celles des ghettomen d'Abidjan. Sylvie Bredeloup montre que les «migrations d'aventures» véhiculent une culture de la mobilité ayant ses territorialités et imaginaires propres.

7 Cet ouvrage est d'une richesse indéniable. Elle nous invite à prendre connaissance de la signification du phénomène migratoire qui a longtemps marqué le destin des populations d'Afrique noire. Après une longue expérience aux côtés de ces "aventuriers» africains, l'auteure nous invite à prendre avec précaution les conséquences qui découlent aujourd'hui de cette pratique migratoire qui, au fur et à mesure, met en péril le destin des hommes et femmes en provenance de l'Afrique noire. Des jeunes de plus en plus motivés par la soif d'aventure, sacrifient leur vie par l'emprunt de pirogues sur la mer dans le seul but d'atteindre l'Europe. Ce phénomène appelé «barça» ou «barsac» (ou la mort) est devenu l'une des questions les plus inhérentes qui alimentent les débats politiques et médiatiques au cours de ces dernières années.

8 Enfin, il faut dire que la démarche adoptée, la diversité des matériaux mobilisés et la rigueur analytique déployée dans cet ouvrage, présentent un grand intérêt pour la connaissance des «migrations d'aventure ». Si l'auteure parle des aventurières dans son ouvrage, à l'issue de la lecture, une considération méthodologique nous invite à nous questionner. Ayant une connaissance du terrain africain et des rapports de genre à l'œuvre, ne peut-on pas considérer Sylvie Bredeloup comme une aventurière? Comment cette femme a-t-elle pu approcher les aventuriers? En bref, il s'agit d'un ouvrage porteur d'espoirs, qui présente un intérêt en termes de nouvelles voies de recherche pour tous ceux qui souhaitent une meilleure compréhension et appréhension du phénomène migratoire. 


\section{BIBLIOGRAPHIE}

Appadurai, Arjun (2005) Après le colonialisme : les conséquences culturelles de la globalisation, Paris, Payot, $326 \mathrm{p}$.

Bertoncello, Barbara; Bredeloup Sylvie (2004) Colporteurs africains à Marseille. Un siècle d'aventure, Paris, Autrement, 166 p. (Français d'ailleurs, peuple d'ici).

De Latour, Eliane (2001) Métaphores sociales dans les ghettos de Côte d'Ivoire, Autrepart, $\mathrm{n}^{\circ}$ 18, pp. 151-176.

Gandoulou, Justin-Daniel (1984) Entre Paris et Bacongo, Paris, Centre de création industrielle, 213 p. (Alors ; $n^{\circ} 3$ ).

Gandoulou, Justin-Daniel (1989) Dandies à Bacongo. Le culte de l'élégance dans la société congolaise contemporaine, Paris, L'Harmattan, 239 p.

Rousseau, Cécile ; Said, Taher M. ; Gagné, Marie-Josée ; Bibeau, Gilles (2001) Rêver ensemble le départ. Construction du mythe chez les jeunes Somaliens réfugiés, Autrepart, $n^{\circ} 18$, pp. 51-68.

Halbwachs, Maurice (1999) La mémoire collective. Paris, Albin Michel, 204 p.

Sartre, Jean-Paul (1972) La Nausée, Paris, Gallimard, 247 p.

\section{NOTES}

1. cf. Sizaire, Laure (2016) S'ouvrir au marché matrimonial globalisé : le cas des femmes russophones, e-Migrinter, $\mathrm{n}^{\circ} 14$.

\section{AUTEURS}

\section{BAKARY DIAKHITÉ}

Doctorant en géographie MIGRINTER - UMR 7301, CNRS/Université de Poitiers

bakary.diakhite@univ-poitiers.fr 